\title{
RTI Execution Environment Using Open Grid Service Architecture ${ }^{\star}$
}

\author{
Ki-Young Choi, Tae-Dong Lee, and Chang-Sung Jeong ${ }^{\star \star}$ \\ School of Electrical Engineering in Korea University, \\ 1-5ka, Anam-Dong, Sungbuk-Ku, Seoul 136-713, Korea \\ $\{2 \times x 195$, lyadlove $@$ snoopy.korea.ac.kr \\ csjeong@charlie.korea.ac.kr
}

\begin{abstract}
HLA(High Level Architecture) has been developed to promote interoperability and reusability within the modeling and simulation community. RTI(Run Time Infrastructure) is a software implementation of HLA which is composed of three components: libRTI, FedExec, and RTIExec. However, the previous RTI has an unfriendly execution environment with static and manual resource allocation and execution. In the paper, we present a RTI execution environment using OGSA(Open Grid Service Architecture) which addresses the problems, so called RTI-G. It supports easy-to-useness, transparency and performance by providing users with dynamic resource allocation and automatic execution. Moreover, it provides an unified view of RTI and Grid services by integrating them as web services on OGSA.
\end{abstract}

\section{Introduction}

Running a large-scale distributed simulation may need a large amount of computing resource residing in geographically different locations. HLA(High Level Architecture) 1 provides application developers with a powerful framework for reuse and interoperability of distributed simulation. However, RTI(Run Time Infrastructure), the implementation of HLA, was not designed to support software applications that need to integrate instruments, displays, computational and information resources managed by diverse organizations. Moreover, the existing RTI(Run Time Infrastructure) has an unfriendly execution environment with static and manual resource allocation and execution. Therefore, the execution of RTI was a painful manual process. Recently, Grid technology has been introduced to address the resource allocation and execution problems in distributed virtual organizations. In the paper, we present a RTI execution environment using OGSA(Open Grid Service Architecture) 2, so called RTI-G, which supports an easy-to-use, transparent execution RTI environment while achieving

\footnotetext{
* This work has been supported by KIPA-Information Technology Research Center, University research program by Ministry of Information \& Communication, and Brain Korea 21 projects in 2005.

** Corresponding author.
} 
high performance by providing users with dynamic resource allocation and automatic execution using various services such as MDS, GRAM, and GridFTP in Globus toolkit 3(GT3) 3] implemented based on OGSA. MDS(Metacomputing Directory Service) provides a standard mechanism for publishing and discovering resource status and configuration information, and GRAM(Globus Resource Allocation Manager) executes the executables in the allocated resource. GridFTP is used to transfer I/O files and executables into the remote resources.

In section 2, we describe the design of RTI-G based on OGSA, and in section 3 , the experimental result. In section 4 , we give a conclusion.

\section{Design of RTI-G}

RTI-G is a grid-enabled implementation of RTI. That is, using services in GT3 based on OGSA, each service of RTI-G is designed to provide automatic and dynamic execution environment. The architecture of RTI-G consists of two layers: RTI service layer and Grid layer. In this section, we describe about each layers respectively more in detail.

\subsection{RTI Service Layer}

RTI layer is composed of three services: libRTI-G, FedExec-G, RTIExec-G. RTI software can be executed on a stand alone workstation or executed over an arbitrarily complex network. RTIExec-G manages the creation and destruction of federation. Each federation is characterized by a single and global FedExec-G, which manages federates joining and resigning the federation. libRTI-G provides RTI service interface to federate developers. The whole simulation in HLA is accomplished through the interaction among libRTI-G, RTIExec-G, and FedExec-G.

OGSA integrates key Grid technologies with Web services mechanisms to create a distributed system framework based on OGSI(Open Grid Services Infrastructure). Each RTI service is defined as a grid service using WSDL(Web Service Description Language) in OGSA to be efficiently incorporated in the Grid environment, and hence to be accessible by standard Grid clients.

Using WSDL, a grid service is defined about how a client interacts with its service instance. That is, the Grid service description is embodied in the serviceType of the instance, along with its associated serviceTypes, portTypes, serviceDataDescriptions, messages, and types definitions. Therefore, in order to be compatible with the grid services, each service in RTI layer should be specified in WSDL. There are two methods for describing the service of RTI layer in WSDL. The first is to write the service in WSDL directly, which is the most versatile method, providing the total control over the description of service PortType. However, it is not user-friendly because WSDL is a rather verbose language. The second is to generate WSDL from an interface language. For example, we can generate WSDL automatically from a Java interface or an IDL interface, which is the easiest method, but not versatile, because very complicated interfaces are not always converted correctly to WSDL. Since our 
RTI is implemented using $\mathrm{C}++$ language, we choose the first method to easily convert our RTI in $\mathrm{C}++$ into WSDL.

We deploy the services in RTI service layer onto GT3 container using GWSDL and WSDD(Web services Deployment descriptor) so that they can be used identically as various other grid services defined on OGSA, thus providing an unified environment of RTI and Grid services. Using the service browser, we can invoke RTI service, and create a service instance as follows: First, user request a service by sending its GSH(Grid Service Handle) to the RTI service factory, which creates the instance. A GSH is a stable name for a Grid service, but does not allow client to actually communicate with the Grid service. RTI service factory maps GSH to GSR(Grid service Reference), a WSDL document which describes how a client communicate with the Grid service.

\section{$2.2 \quad$ Grid Layer}

Grid layer comprises several services such as MDS, GRAM, and GridFTP implemented in GT3. MDS is used to allocate the resource with high performance by monitoring resource status and configuration information, and GRAM to execute the RTI service in the allocated resource. GridFTP is used to transfer the RTI service executables into the remote resources.

\section{$3 \quad$ Experiments}

For the evaluation of RTI-G, we execute Nexio, a networked shooting game on RTI-G and RTI respectively, and compare their performance. In Nexio, two players can play shooting game, and communicate through services in RTI. For each case, we measure the data transfer rate between players. The data size of each object in the game is 10 Kbytes, and with the advancement of each stage, the number of objects is increased by 100. In the first case, player 1 requests the join into a federation, and then RTIExec and FedExec are created subsequently on the server specified by player 1 . Then, player 2 in different host joins into the federation as in figure 1(a). In the second case, player 1 requests the join into a federation, and then RTIExec and FedExec are created in the server allocated by RTI service factory after accessing MDS and selecting the one with the best performance as in figure 1(b). RtiExec service is created as RTIservice instance, and makes FedExec service is generated through fork operation on the allocated server. After that, player 2 joins into the federation, and they exchange information about objects in the game.

We used each client machines with same specification to reduce side effects according to different computing power of each machine. All client host has $850 \mathrm{MHZ}$ CPU, 256MB memory, 512MB of swap, running under the Linux OS. There are three servers with different specs as shown in figure 1(c). In the second case, the best server is selected using MDS by RTI service factory. As we see in figure $1(\mathrm{~d})$ which shows the data transfer rate vs total object size, RTI-G has the better performance than RTI. 

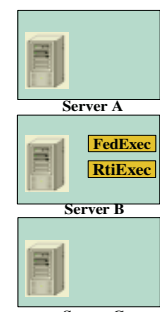

(a)

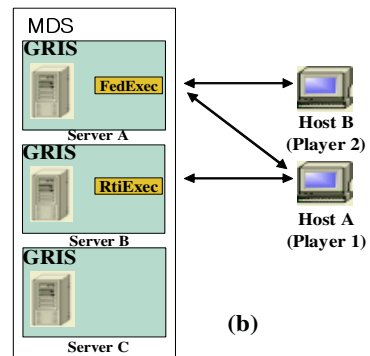

\begin{tabular}{|c|c|c|c|c|c|}
\hline $\begin{array}{c}\text { Machine } \\
\text { Name }\end{array}$ & $\begin{array}{c}\text { Model } \\
\text { Name }\end{array}$ & $\begin{array}{c}\text { CPU } \\
\text { MHz }\end{array}$ & $\begin{array}{c}\text { Total } \\
\text { Memory } \\
(\text { MB) }\end{array}$ & $\begin{array}{c}\text { Swap } \\
\text { Memory } \\
(\text { MB) }\end{array}$ & OS \\
\hline Host A & $\begin{array}{c}\text { Pentium } \\
\text { III }\end{array}$ & 850 & 256 & 512 & Linux 2.4 \\
\hline Host B & $\begin{array}{c}\text { Pentium } \\
\text { III }\end{array}$ & 850 & 256 & 512 & Linux 2.4 \\
\hline \hline $\begin{array}{c}\text { Machine } \\
\text { Name }\end{array}$ & $\begin{array}{c}\text { Model } \\
\text { Name }\end{array}$ & $\begin{array}{c}\text { CPU } \\
\text { MHz }\end{array}$ & $\begin{array}{c}\text { Total } \\
\text { Memory } \\
\text { (MB) }\end{array}$ & $\begin{array}{c}\text { Memory } \\
\text { (MB) }\end{array}$ & OS \\
\hline Server A & $\begin{array}{c}\text { Pentium } \\
\text { IV }\end{array}$ & 1700 & 512 & 1024 & Linux 2.4 \\
\hline Server B & $\begin{array}{c}\text { Pentium } \\
\text { IV }\end{array}$ & 1500 & 256 & 512 & Linux 2.4 \\
\hline Server C & $\begin{array}{c}\text { Pentium } \\
\text { IV }\end{array}$ & 1500 & 512 & 512 & Linux 2.4 \\
\hline \multicolumn{5}{|c|}{ (c) }
\end{tabular}

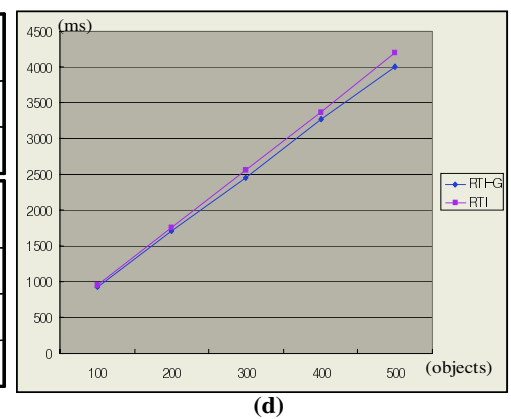

Fig. 1. (a)The first case using RTI, (b)The second case using RTI-G, (c) Resource performance, (d) Comparison of data transfer rate on RTI and RTI-G

\section{Conclusion}

In the paper, we have presented RTI-G which supports an easy-to-use, transparent execution RTI environment while achieving high performance by providing users with dynamic resource allocation and automatic execution using various services in GT3. The specification of RTI-G in GWSDL and the deployment of RTI-G services in RTI service layer onto GT3 container provide an integrated unified environment of RTI and Grid services, thus supporting easy-to-use, transparency, and performance. Also, we have described the experimental result for RTI-G by comparing it with RTI. As a future work, we are designing a RTI portal for the efficient execution and performance monitoring of RTI with application to distributed war game simulation.

\section{References}

1. IEEE Standard for Modeling and Simulation,"High Level Architecture (HLA) Federate Interface Specification," IEEE Std 1516.1-2000 54.

2. D.Talia, "The Open Grid Services Architecture: where the grid meets the Web," Internet Computing, IEEE, Volume: 6 , Issue: 6, Nov.-Dec., pp.67-71, 2002.

3. I. Foster, C. Kesselman, "Globus: A Metacomputing Infrastructure Toolkit, Intl J. Supercomputer Applications," 11(2), pp. 115-128, 1997. 